\title{
Análisis de las barreras para la adherencia terapéutica en mujeres colombianas con VIH/sida: cuestión de derechos de salud
}

\author{
Marcela Arrivillaga-Quintero, PhD. (I)
}

\begin{abstract}
Arrivillaga-Quintero $M$. Análisis de las barreras para la adherencia terapéutica en mujeres colombianas con $\mathrm{VIH} /$ sida: cuestión de derechos de salud. Salud Publica Mex 2010;52:350-356.
\end{abstract}

\begin{abstract}
Resumen
Objetivo. Identificar y analizar barreras para la adherencia terapéutica de mujeres colombianas con VIH/sida. Material y métodos. Estudio cualitativo, descriptivo-interpretativo, con análisis de contenido conducido entre los años 2008 y 2009. Las participantes fueron 66 mujeres de la ciudad de Cali, con quienes se efectuaron cinco grupos focales. Se desarrollaron también entrevistas semiestructuradas con siete informantes clave. Resultados. Las principales barreras están determinadas estructuralmente por el sistema de salud vigente en Colombia, basado en el mercado de aseguramiento. Las mujeres ven afectados sus derechos al tratamiento oportuno y continuo, a la confidencialidad y a la no discriminación, y a la atención integral con enfoque de género, por lo que la adherencia terapéutica se ve afectada. Conclusiones. El sistema de salud vigente en Colombia determina procesos críticos que afectan la adherencia de mujeres con $\mathrm{VIH} / \mathrm{sida}$. No obstante, debe conducirse más investigación para identificar asociaciones y relaciones específicas con los sistemas de salud no universales.
\end{abstract}

Palabras clave: adhesión al tratamiento; $\mathrm{VIH}$; sida; mujeres; derechos humanos; Colombia
Arrivillaga-Quintero M.

Analysis of barriers to therapeutic adherence

for Colombian women with HIVIAIDS:

a question of health rights.

Salud Publica Mex 2010;52:350-356.

\section{Abstract}

Objective. To identify and analyze HIVIAIDS treatment adherence among Colombian women. Material and Methods. A qualitative, descriptive-interpretative study with content analysis was developed between 2008 and 2009. Sixty six women participated in five focus group discussions. To complement data, semi-structured interviews with seven key informants were conducted. Results. Main barriers are determined structurally by the current Colombian health system, based on insurance market. The right to access to treatment in a timely and continuous manner, the rights to confidentiality and non discrimination, and the right to quality care with gender focus are affected among participant women. Consequently, adherence is negatively affected. Conclusions. The current Colombian health system determines critical processes affecting HIVIAIDS treatment adherence in women. However, further research must be conducted to identify specific associations with non-universal health systems.

Key words: medication adherence;HIV;AIDS; women; human rights; Colombia 
$\mathrm{E}^{1}$ actual sistema de salud colombiano fue instaurado mediante la Ley 100 de 1993. El sistema se estructuró acogiendo las recomendaciones del Banco Mundial en la década de los noventa, que promovía la idea de que los derechos universales a cargo de recursos públicos eran inviables económicamente, y que la estrategia de focalización y subsidio a la demanda era la más adecuada para la atención de la población pobre. Con esta lógica se desarrolló un sistema basado en el mercado de aseguramiento individual como mecanismo para disminuir el gasto público en salud. En este modelo la distribución de servicios de salud se realiza acorde con la noción de aseguramiento de riesgos en vez de la noción de necesidades de la población. ${ }^{1}$

Actualmente, el sistema cuenta con tres formas de afiliación: régimen contributivo, régimen subsidiado y vinculados. Los afiliados al régimen contributivo son las personas con capacidad de pago. Los afiliados al régimen subsidiado son personas sin capacidad de pago, previamente identificadas como beneficiarias del subsidio. Los vinculados son aquellas personas en situación de pobreza que no se encuentran formalmente afiliadas a ningún régimen, y que mientras logran ser beneficiarias del subsidio, deben ser atendidas en instituciones que tengan contrato con el Estado. En este sistema la población enfrenta "itinerarios burocráticos" ${ }^{2}$ caracterizados por retrasos y negaciones para acceder a los servicios, con consecuencias como la prolongación del sufrimiento, complicaciones de salud, discapacidad y muerte. El incremento en el número de colombianos asegurados después de la Ley 100 contrasta con lo que se ha llamado la judicialización del sistema, ya que se interponen cientos de acciones legales (tutelas) para garantizar el derecho a la salud. No en vano, el reciente informe de la Defensoría del Pueblo publicado en 2009 concluye que la tutela continúa siendo el mecanismo más utilizado por los colombianos para hacer valer sus derechos de salud, y que $78.1 \%$ de las tutelas se interponen contra las entidades promotoras de salud (EPS) que administran los regímenes contributivo y subsidiado.

En este contexto, el VIH/sida continúa siendo una problemática nacional. Se calcula que $11 \%$ de las personas que viven con VIH no tienen seguro de salud y no hay datos disponibles sobre 55\% de la población afectada. El acceso a la terapia antirretroviral (TAR) también es limitado, estimado para 2007 en 38\% de cobertura. ${ }^{3,4}$ Las barreras de acceso al sistema de salud son mayores para las mujeres en situación de pobreza, quienes tienen acceso insuficiente a los seguros de salud, invierten mayores recursos propios como fuente de financiación, y se exponen a atención de baja calidad por la vía del subsidio. ${ }^{5,6}$
Teniendo en cuenta el escenario descrito, este estudio tuvo como objetivo identificar y analizar barreras para la adherencia terapéutica de mujeres colombianas con VIH/sida. Acogiendo la propuesta de Naciones Unidas, la perspectiva de derechos humanos, en especial del derecho a la salud, es el marco de análisis para los hallazgos que se presentan. Desde esta perspectiva, se reconoce la relación intrínseca entre la salud y el concepto de bienestar que recoge la dimensión individual y colectiva del derecho a la salud. Se analizan los resultados considerando el derecho a la salud como "un derecho al disfrute de toda gama de facilidades, bienes, servicios y condiciones necesarios para alcanzar el más alto nivel posible de salud." ${ }^{7}$

\section{Material y métodos}

Estudio cualitativo de tipo descriptivo-interpretativo, aprobado por los comités de ética de la Pontificia Universidad Javeriana Cali y de las instituciones donde se recolectó la información, previa firma de consentimiento informado. Las participantes fueron 66 mujeres diagnosticadas con $\mathrm{VIH} /$ sida de la ciudad de Cali. Los criterios de inclusión fueron: estar en TAR, mayores de 18 años y no gestantes. Las mujeres fueron contactadas por medio de las instituciones de salud e invitadas a formar parte del estudio mediante llamadas telefónicas.

Grupos focales. Se efectuaron cinco grupos focales; aproximadamente 13 mujeres participaron en cada grupo. El principal tema de discusión fue: "Barreras para la adherencia terapéutica". Los temas específicos fueron: acceso al tratamiento, calidad de la atención recibida y necesidades de atención, cumplimiento de citas médicas y régimen farmacológico, confidencialidad en las instituciones de salud, estigma y discriminación por parte de los proveedores de salud, barreras de acceso geográfico y gasto de bolsillo en salud. Durante el desarrollo de los grupos focales un observador participante tomó notas de campo. Se realizaron grupos focales hasta completar el criterio de saturación teórica.

Entrevistas semiestructuradas. Se desarrollaron entrevistas semiestructuradas con siete profesionales de la salud de distintas instituciones de la ciudad de Cali. Su experiencia promedio de trabajo con personas con $\mathrm{VIH}$ / sida fue de 10 años. Las entrevistas contenían cinco preguntas que cubrían los temas de: adherencia terapéutica en mujeres con VIH/sida, barreras para la adherencia, estigma y discriminación, atención integral, relación entre tipos de afiliación al sistema de salud y adherencia.

Análisis de datos. Tanto los grupos focales como las entrevistas semiestructuradas fueron grabados en audio 
para su posterior transcripción y análisis. Se realizó análisis de contenido, ${ }^{8}$ utilizando el software Atlas Ti. El procedimiento siguió las siguientes etapas: 1) lectura sistemática de la información; 2) deconstrucción de los datos, reduciendo la información a 331 citaciones agrupadas en 65 códigos; 3) análisis cuantitativo para determinar la frecuencia de aparición de los códigos, y 4) reconstrucción y síntesis de la información para obtener finalmente tres categorías interpretativas con sus correspondientes subtemas. En todo momento se tuvieron en cuenta las notas de campo como complemento para analizar y validar la información.

\section{Resultados}

Características de las participantes. La edad promedio de las mujeres participantes fue de 36 años. De ellas, 52\% fueron casadas y en unión libre, $22 \%$ solteras, $15 \%$ separadas o divorciadas y $11 \%$ viudas. En cuanto a estrato socioeconómico, $73 \%$ pertenecieron a los estratos 1,2 y 3 (bajo y medio-bajo en la clasificación oficial colombiana) y $27 \%$ a los estratos 4,5 y 6 (medio-alto y alto); $34 \%$ contaron con escolaridad primaria, $47 \%$ con estudios de secundaria y $19 \%$ con estudios universitarios. Por su afiliación, $48 \%$ resultaron afiliadas al sistema de salud en el régimen contributivo, $42 \%$ en el régimen subsidiado y $10 \%$ se encontraron en la categoría de vinculadas. El $34 \%$ de las participantes fueron mujeres cabeza de familia y responsables económicamente de sus hogares. A continuación se describen los resultados en las tres categorías interpretativas resultantes, y bajo el marco de los derechos en salud.

I. Derecho al tratamiento oportuno y continuo: condición necesaria para la adherencia. Si bien este estudio incluyó mujeres en TAR, muchas de ellas enfrentaron barreras de acceso cuando fueron diagnosticadas, lo que afectó tanto la oportunidad como la continuidad del tratamiento. Las mujeres que en algún momento no tenían ningún tipo de aseguramiento, consideradas como población vinculada, tuvieron serias dificultades para acceder a la TAR. Estas mujeres estuvieron en listas de espera y finalmente acudieron a mecanismos legales como la acción de tutela para obtener el tratamiento, asesoradas por organizaciones no gubernamentales para la defensa de personas con $\mathrm{VIH} /$ sida.

"Es muy horrible, demoraron mis medicamentos. Que para mañana, pasado mañana, yo dije nooo!!, es que a mí me tienen que entregar mis medicamentos, como me van a decir tres, cuatro, cinco meses, que no están, que venga mañana. Menos mal yo tengo mi reserva de medicamentos, porque es que como uno no sabe con que le van a salir." (Mujer, 37 años, vinculada.)

"Las mujeres que no tienen aseguramiento, tendrían que comprar el tratamiento porque la entidad territorial [el Estado] que atiende a la población pobre no asegurada, no lo otorga a tiempo. El que debe garantizar el tratamiento es el Departamento [Gobernación]. Sin embargo, en muchas ocasiones no logran obtener el tratamiento, a las mujeres les toca estar en listas de espera o tienen que poner tutela [acción legal] para que les den la TAR". (Profesional de la salud.)

Por su parte, las mujeres aseguradas expresaron sus problemáticas para el acceso estable y continuo al tratamiento, debidas a cambios en su condición laboral, desempleo suyo o de sus parejas, de quienes dependen, $\mathrm{y}$ regulaciones de las aseguradoras. Tienen problemas relacionados con periodos mínimos de cotización y con procesos de selección adversa cuando se obstaculiza el traslado a otra aseguradora estando diagnosticadas con $\mathrm{VIH} /$ sida. Evidencia de la inestabilidad en el acceso al tratamiento es que las mujeres tienen, como práctica común, mantener una reserva de los medicamentos antirretrovirales, incluso por recomendación directa de los médicos que las atienden.

"Al comienzo lo más difícil era acceder al tratamiento cuando estaba en [ $\mathrm{x}$ aseguradora], de allí me retiré porque no me daban el tratamiento, tuve muchísimas dificultades. Me pasé a [x aseguradora], ya me iban a devolver, que porque yo iba con el diagnóstico. Entonces allí me tocó que empezar a poner tutelas [acción legal], entonces pues ya tenía más conocimientos y empecé a defender mis derechos." (Mujer, 28 años, régimen contributivo.)

"A mi casi no me dan el tratamiento, me tocó que ir a hacer fila y madrugar muchas veces a la Secretaría de Salud para que me dieran el carnet, de ahí fui al hospital y finalmente después de cuatro meses me dieron la cita y me atendieron." (Mujer, 42 años, régimen subsidiado.)

De acuerdo con los profesionales entrevistados, el tipo de afiliación al sistema de salud afecta la adherencia de las mujeres. Mujeres vinculadas o en el régimen subsidiado presentan menor adherencia en comparación con mujeres en el régimen contributivo o con seguros privados de salud.

“El tipo de afiliación al sistema de salud incide en la adherencia. Para muchas mujeres el no tener aseguramiento 
se suma a la angustia de estar diagnosticadas y no tener la posibilidad de tratarse; además, muchas veces no tienen dinero para los costos como movilizarse y pagar los exámenes. Esto es peor en mujeres que vienen de áreas rurales." (Profesional de la salud.)

Las mujeres recurren frecuentemente a mecanismos legales para acceder y mantener el tratamiento. Las mujeres más pobres generalmente requieren asesoría de ONG, mientras las mujeres de posiciones sociales más altas tienen conocimiento, recursos y habilidades personales, que les permitieron realizar el trámite por sí mismas. Las mujeres que defienden sus derechos como usuarias del servicio de salud tienen tendencia a presentar mejor nivel de adherencia.

"Yo tuve dificultades, porque no me entregaban los medicamentos ARV, entonces eso me desgataba. Me retiré de esa EPS [x aseguradora], y me pasé para otra. Llegué allá y también tuve dificultades, pero después yo ya conocía más la parte legal, entonces empecé a hacer tutelas, desacatos, derechos de petición [acciones legales]. Aprendí que cuando uno no tiene conocimiento pues a uno le parece suficiente con que le den un poquito, como si le estuvieran haciendo un favor, jes que uno tiene derecho! Menos mal allá en la fundación [ONG a la cual asiste] le ayudan mucho a uno para defender sus derechos, tener el tratamiento y tomárselo." (Mujer, 41 años, régimen subsidiado.)

"Aquí en la Fundación prestamos asesoría legal para que las personas con VIH tengan sus medicamentos a tiempo. Las mujeres con mejor adherencia, y curiosamente las del régimen contributivo son las más interesadas en poner rápido las tutelas. A las mujeres más pobres les cuesta mucho trabajo y hay que acompañarlas más en el proceso." (Profesional de la salud.)

Además de las barreras en el acceso, las mujeres perciben obstáculos para su adherencia impuestos por la tramitología y la fragmentación de los servicios. Múltiples visitas a las instituciones de salud en diferentes momentos son necesarias para las citas médicas, para solicitar órdenes médicas y para reclamar los medicamentos. Las mujeres que viven en zonas rurales son las más afectadas. Esta tramitología supone obstáculos a las mujeres empleadas para pedir permisos laborales y representa un gasto de bolsillo que afecta el presupuesto familiar. Las mujeres pobres que realizan un sobreesfuerzo económico y pagan su afiliación al régimen contributivo sólo para acceder al antirretroviral no cuentan con ingresos y estabilidad económica para cubrir gastos extras.
“... uno va a la cita, luego le toca que devolverse para que se los autoricen [los medicamentos], luego ir a que le den la orden y luego ir a que se los entreguen, entonces hay algunos que no le gusta, es que a ninguno nos gusta. Por eso es que uno piensa que si hubiese una forma más fácil sería mejor. Si sólo fuera ir al médico y que saliera de ahí y le entregaran los medicamentos. Yo porque tengo reservas, si no las tuviera pararía mis actividades para ir por mis medicamentos, pero otras mujeres no, muchas de las mujeres no trabajan, a veces uno no sabe qué es mejor, porque las que trabajan les ponen los 'peros' los jefes y las que no trabajan no tienen con qué ir al médico." (Mujer, 40 años, vinculada.)

II. Derecho a la confidencialidad y a la no discriminación: ambiente propicio para la adherencia. En general, las mujeres piensan que el VIH/ sida es una "enfermedad" de la que deben avergonzarse; consideran que para la sociedad no es lo mismo referirse a otra enfermedad, así sea mortal, que referirse al VIH/ sida. Al mismo tiempo, tienen una fuerte convicción sobre la discriminación, el rechazo y la lástima que produce el diagnóstico, basándose en el estigma social asociado al mismo. Temen que su familia conozca su estado de salud y se rompa la imagen que se ha construido sobre ellas, incluyendo que sus prácticas sexuales se verían cuestionadas. El costo emocional del diagnóstico implica estrés que por momentos les resulta agotador y desgastante.

“Terrible al principio, fue como si la vida se me acabara, yo jamás pensé que una enfermedad como esa me llegaría, siempre la vi como algo que le puede dar a los demás pero no a mí. Al principio no quería vivir, ni hablar con nadie, no quería ver a mi familia, sufrí mucho, por miedos, por sentirme sucia y mala. Yo pensaba que moriría en poco tiempo y no sabía que hacer con tantas cosas pendientes que tenía." (Mujer, 37 años, régimen contributivo.)

De acuerdo con los profesionales de la salud, las mujeres de más altas posiciones sociales experimentan mayor estigma que las mujeres de posición social media o baja. A su vez, temen que se viole la confidencialidad del diagnóstico en las instituciones de salud, y por eso prefieren pagar de forma particular sus consultas médicas para no quedar registradas en el sistema. Estas mujeres no utilizan su servicio de salud así cuenten con afiliación al régimen contributivo.

“En cambio las de la clase alta, nunca van a los centros de salud porque temen ser registradas en el sistema. Buscan ayuda particular, ipero ir a una ONG jamás! Tengo una paciente en mi consulta particular que viaja a otra ciudad 
a tratarse con un médico especialista. Dice que no confía en los médicos, que las instituciones no son muy éticas y que la información de su diagnóstico se puede filtrar." (Profesional de la salud.)

Las mujeres vinculadas o en el régimen subsidiado reportan experiencias de rechazo y prefieren no asistir a un lugar de atención cuando han sido objeto de discriminación, lo que afecta directamente su adherencia. Además, deben rotar por distintos centros de salud, no mantienen un único médico tratante y son atendidas por médicos sin experiencia en el manejo del VIH.

"Eso fue en ese Hospital, porque a nosotros los de [x aseguradora] nos atienden, un tiempo en un hospital, otro tiempo en otro. Eso es de para allá y para acá. Ellos lo miran a uno como un bicho raro, incluso en el personal médico, ahora hay muchos doctores que no saben nada o saben muy poco del VIH. En el hospital me atendieron unos estudiantes. Yo entré al consultorio y ninguno quería examinarme. Entonces me dio tanta rabia, que yo dije: '¿me van a examinar, sí o no?'. Después de eso yo no quería volver por allá." (Mujer, 27 años, régimen subsidiado.)

III. Derecho a la atención integral con enfoque de género. La gran mayoría de las mujeres reportaron ser atendidas por médicos generales, sin o con poco entrenamiento y experiencia en el control del VIH. Esto abiertamente genera desconfianza en su capacidad para orientar el tratamiento y hace que las mujeres recurran a otras consultas especializadas.

"Yo fui donde otro médico especialista en VIH porque en la EPS [x aseguradora] me mandaron donde un médico nuevo y sin experiencia. En la consulta le dije que había hablado con el especialista en VIH que me dijo que lo más conveniente era que yo tomara estos medicamentos porque tengo mutaciones de esta clase, tengo resistencia de esta otra clase, estos no me caen bien. Al principio al médico no le gustó, pero luego entendió que era lo mejor." (Mujer, 39 años, régimen contributivo.)

Además de lo anterior, las mujeres perciben y expresan explícitamente la necesidad de que el sistema de salud desarrolle programas integrales con enfoque de género. Destacan que el reconocimiento a sus necesidades específicas, más allá de la focalización en la atención de gestantes, es fundamental para una adecuada adherencia; incluso, argumentan la importancia de una respuesta intersectorial para atender las condiciones de vida, en especial de las mujeres más pobres.
"Pienso que falta atención integral de verdad para las mujeres con VIH, cuando uno tiene un buen acompañamiento y también apoyo en la parte económica, la adherencia mejoraría. Pero uno pensando que no tiene dinero, que no tiene para una buena alimentación, que no tiene un acompañamiento. Se necesita un programa que vea a la mujer como un ser humano, como mujer, porque nos ven distintas cuando somos gestantes, pero nació el muchachito y ¿que pasó con la mujer? Todo es para las gestantes, pero ¿el resto de las mujeres qué?, estamos de últimas, y eso es lo que pasa, que si está gestante se le abren las puertas en los servicios de salud, pero si el niñito salió, serán niños sanos pero huérfanos." (Mujer, 38 años, régimen subsidiado.)

\section{Discusión}

Los resultados de este estudio muestran que las principales barreras para la adherencia de mujeres con VIH/ sida están determinadas estructuralmente por el sistema de salud vigente en Colombia. Las mujeres ven afectados sus derechos al tratamiento oportuno y continuo, a la confidencialidad y a la no discriminación, y a la atención integral con enfoque de género. En consecuencia, la adherencia terapéutica se ve vulnerada y su riesgo de mortalidad se incrementa.

A nivel mundial, se ha reportado que el acceso a la TAR en los países de ingresos bajos y medios alcanzó casi tres millones de personas para finales del 2007, contribuyendo a la reducción de la mortalidad por $\mathrm{VIH} /$ sida. $^{9}$ No obstante, los hallazgos de este estudio muestran que las mujeres colombianas enfrentan serias barreras de acceso al tratamiento. La más grave situación la viven mujeres pobres, en categoría vinculadas o afiliadas al régimen subsidiado, quienes para poder acceder a la TAR primero deben demostrar su pobreza en un sistema de registro del estado colombiano denominado el SISBEN (sistema de identificación para potenciales beneficiarios de subsidios). Esta barrera estructural impone tiempos de espera, tramitación excesiva, afecta directamente su derecho al tratamiento oportuno, su adherencia terapéutica y su derecho a la salud.

Estudios recientes sobre el acceso continuo de las mujeres a la TAR muestran datos similares a los aquí presentados. Mujeres de bajos ingresos en los Estados Unidos también enfrentan barreras institucionales y acceso limitado, producto del sistema de salud vigente en ese país. Los principales obstáculos se relacionan con altos costos de la atención, elevado gasto de bolsillo e impedimentos de las compañías de seguros para que accedan al tratamiento. ${ }^{10}$ En Colombia, se encontró que las mujeres con $\mathrm{VIH} /$ sida vinculadas o en régimen subsi- 
diado tienen mayor riesgo de presentar baja adherencia terapéutica. ${ }^{11}$ Por el contrario, existe evidencia sobre los beneficios de proveer acceso universal y gratuito a la TAR. Además de ser un mecanismo para garantizar el derecho a la salud, promueve la adherencia terapéutica y favorece resultados positivos del tratamiento en personas viviendo con $\mathrm{VIH} .{ }^{12}$ Se ha demostrado que el diagnóstico temprano, la elegibilidad del tratamiento apropiado y la provisión de TAR gratuita en países de bajos ingresos se asocia con baja mortalidad. ${ }^{13} \mathrm{~A}$ su vez, hay datos que indican que el acceso universal reduce la transmisión del virus y contribuye al control de la epidemia. ${ }^{14}$ En América Latina, la política de acceso a la TAR implementada desde 1996 en el sistema de salud pública brasileño ha tenido efectos positivos en la calidad de vida de la población afectada; han disminuido las tasas de morbi-mortalidad, las admisiones hospitalarias y los costos del tratamiento. En esta región, la experiencia brasileña ha aportado evidencia suficiente para afirmar que una iniciativa de esta naturaleza es costo-efectiva y económicamente viable. ${ }^{15}$

En cuanto al derecho a la confidencialidad y a la no discriminación, los hallazgos mostrados son acordes con los planteamientos de otros autores. Las mujeres con VIH son una población estigmatizada y objeto de rechazo a raíz del diagnóstico; pueden sufrir más discriminación en comparación con los hombres y tienen dificultades en sus relaciones con los proveedores de salud. ${ }^{16}$ Aquí se han presentado formas comunes de discriminación ya reportadas en la literatura, como por ejemplo el abierto rechazo o la posible violación a la confidencialidad. El estigma y el temor a revelar el estatus de salud representan barreras importantes para la adherencia y para la atención integral. ${ }^{17}$ De acuerdo con informes gubernamentales, sólo un tercio de los países tiene leyes o utiliza indicadores de resultados para la reducción del estigma y la discriminación. ${ }^{9} \mathrm{La}$ mayoría de los países, incluido Colombia, deben realizar mayores esfuerzos prácticos en este sentido y proteger el derecho a la confidencialidad y a la no discriminación de las personas que viven con VIH. ${ }^{12}$ Los hallazgos de este estudio confirman la necesidad de que los estados tomen medidas legislativas, presupuestales, judiciales y promocionales para tal propósito.

Finalmente, los resultados ponen en evidencia que el derecho a la atención integral resulta vulnerado para las mujeres, aun ante la evidencia científica que indica cómo el acceso a la seguridad social, el cuidado primario, recibir siempre el tratamiento por parte del mismo proveedor de salud y recibir atención de especialistas, contribuyen a una mayor adherencia en $\mathrm{VIH} /$ sida. $^{18}$ Como han planteado otros autores, optimizar la adherencia requiere que los programas de TAR se integren con otros servicios; la atención integral de la mujer con VIH debe incluir educación, apoyo psicológico, intervención familiar, promoción de grupos de apoyo, soporte nutricional ${ }^{19} \mathrm{y}$ acceso a los servicios de salud sexual y reproductiva. ${ }^{20}$ Además, los datos indican que la atención integral tiene efectos positivos en la supresión de la carga viral. ${ }^{21}$

La evidencia mostrada en este estudio indica que a nivel nacional Colombia incumple la reglamentación sobre la atención por parte de las instituciones de salud a las personas con VIH/sida, establecida en el Decreto 1543 de 1997. A nivel internacional, el derecho a la salud se ve vulnerado e incumple la suscripción de varios pactos internacionales para la defensa de los derechos de las personas con VIH. La defensa del derecho a la salud de las mujeres colombianas afectadas por el virus y la generación de condiciones para facilitar su adherencia requiere promover la equidad de género mediante más y mejores políticas públicas. ${ }^{11,22}$ Se hace necesario aumentar su bienestar e independencia económica, generar mejores ingresos y defender su derecho al trabajo para una vida digna. Desafortunadamente, Colombia es uno de los países de América Latina que se encuentran entre las puntuaciones más bajas en la generación de políticas relacionadas con la mujer y la equidad de género. ${ }^{9}$ Además, como han sugerido otros autores, no luce admisible que una política pública como la seguridad social en salud se fundamente en la economía de mercado ${ }^{23}$ y desconozca la realidad social de millones de personas afectadas, que incluyen a las mujeres. Los hallazgos ilustran la forma en que las condiciones del sistema de salud representan procesos críticos de determinación general y estructural de la adherencia de mujeres colombianas con $\mathrm{VIH} /$ sida. No obstante, con el propósito de avanzar en el conocimiento de la determinación social de la adherencia, debe conducirse una cantidad mayor de investigación cualitativa y cuantitativa para identificar asociaciones y relaciones específicas con los sistemas de salud no universales.

\section{Referencias}

I.Vega R, Hernández A. Evaluación de los efectos de la política de focalización de subsidios en salud sobre la población sin capacidad de pago de cuatro localidades de Bogotá, D.C., Colombia, 200I. Rev Salud Publica 2003;5(3):284-300.

2.Abadia CE, Oviedo DG. Bureaucratics itineraries in Colombia.A theoretical and methodological tool to assess managed-care health care system. Soc Sci Med 2009;68(6): I I53-I I 60.

3. García Bernal R, Luque Núñez R, Mcdouall Lombana J, Moreno Díaz LA. Infección por vih y sida en Colombia. Estado del arte 2000-2005. Bogotá: ONUSIDA Colombia / Ministerio de la Protección Social de Colombia, 2006. 
4.WHO, UNAIDS, UNICEF. Epidemiological Fact Sheet on HIV and AIDS. Core data on epidemiology and response. Colombia 2008 Update. Geneva: UNAIDS/WHOWorking Group on Global HIVIAIDS and STI Surveillance, 2008.

5. Ewig C, Hernández A. Gender equity and health sector reform in Colombia: Mixed state-market model yields mixed results. Soc Sci Med 2009;68(6): II I45-II52.

6. Guanizo-Herreño C,Agudelo C. Equidad de género en el acceso a los servicios de salud en Colombia. Rev Salud Pública 2008;10(I):44-57.

7. Organización de las Naciones Unidas. Comité de Derechos Económicos, Sociales y Culturales. Observación General No. I4. El derecho al disfrute del más alto nivel posible de salud. Ginebra: ONU, 2000.

8. Deslauriers JP. Recherche qualitative: Guide pratique. Montréal: McGraw Hill, I99I.

9. ONUSIDA. Informe sobre la epidemia mundial del sida, 2008. Resumen de orientación. Ginebra: ONUSIDA, 2008.

10. Stevens PE, Keigher SM. Systemic barriers to health care access for U.S. women with HIV:The role of cost and insurance. Int J Health Serv 2009;39(2):225-243.

I I.Arrivillaga M, Ross M, Useche B,Alzate ML, Correa D. Social position, gender role, and treatment adherence among Colombian women living with HIV/AIDS: social determinants of health approach. Panam J Public Health 2009;26(6):502-5I0.

12. World Health Organization/UNAIDS. Progress on global access to HIV antiretroviral therapy. Switzerland:WHO, 2006.

13. The Antiretroviral Therapy in Lower Income Countries (ART-LINC) Collaboration,ART Cohort Collaboration (ART-CC) groups. Mortality of HIV-I-infected patients in the first year of antiretroviral therapy: comparison between low-income and high-income countries. Lancet 2006;367:8I7-824.
14. Chi-Tai F, Hsu-Mei H, Shiing-Jer T, Mao-Yen C,Yu-Yin C, Jing-Shiang H, et al. Decreased HIV transmission after a policy of providing free access to highly active antiretroviral therapy in Taiwan.J Infect Dis 2004;190:879-885. 15. Teixeira PR,Vitória MA, Barcarolo J.Antiretroviral treatment in resource-poor settings: the Brazilian experience. AIDS 2004; 18:S5-S7. 16. Ruiz-Torres Y, Cintrón-Bou F,Varas-Diaz N. AIDS-related stigma and health professionals in Puerto Rico. Interamerican J Psychology 2007;4I(I):49-56.

17. Murray LK, Semrau K, McCurley E, Thea D, Scott N, Mwiya M, et al. Barriers to acceptance and adherence of antiretroviral therapy in urban Zambian women: a qualitative study. AIDS Care 2009;2I(I):78-86. 18. Burke-Miller JK, Cook JA, Cohen MH, Hessol NA, Wilson TE, Richarson JL, et al. Longitudinal relationships between use of highly active antiretroviral therapy and satisfaction with care among women living with HIVIAIDS.Am J Public Health 2006;96(6): I044-I05I.

19. Cotrim A, Dias M, Duarte S. Evaluation of the care of women living with HIV/AIDS in Sao Paulo Brazil.Aids Patient Care and STDs 2003; I7(2):85-93.

20. Makwiza I, Nyirenda L, Bongololo G, Banda T, Chimzizi R, Theobald $S$. Who has access to counseling and testing and anti-retroviral therapy in Malawi - an equity analysis. Int J Equity Health 2009;8:I3. doi:10.1 I86/1475-9276-8-13.

21. Hoang T, Goetz MB, Yano EM, Rossman B, Anaya, HD, Knapp H, et al. The impact of integrated HIV care on patient health outcomes. Med Care 2009;47(5):560-567. doi:I0.1097/MLR.0b0I3e3I819432a0.

22.Arrivillaga M,Alzate ML, Useche B. Políticas públicas, sistema de salud y mujeres con VIH/Sida en Colombia: Un análisis crítico. Rev Gerenc Polit Salud 2009;8(I6):58-7I.

23. Cardona A, Mejía L, Nieto E, Restrepo R. Temas críticos en la reforma de la Ley de seguridad social de Colombia en el capítulo de salud. Rev Fac Nac Salud Publica 2005;23(I): I 17-I33. 